\title{
Proteolytic activity of Crocodylus porosus transthyretin protease and role of the terminal polypeptide sequences
}

\author{
Ladda Leelawatwattana ${ }^{\mathrm{a}}$, Verayuth Praphanphoj ${ }^{\mathrm{b}, \mathrm{c}}$, Porntip Prapunpoj ${ }^{\mathrm{a}, *}$ \\ a Department of Biochemistry, Faculty of Science, Prince of Songkla University, Hat Yai, \\ Songkhla 90110 Thailand \\ b The Centre of Medical Genetics, Rajanukul Institute, Din-Daeng District, Bangkok 10400 Thailand \\ c Medical Genetics Centre, Sai Mai District, Bangkok 10220 Thailand
}

${ }^{*}$ Corresponding author, e-mail: porntip.p@psu.ac.th

Received 31 Jul 2015

Accepted 6 Jun 2016

\begin{abstract}
Human transthyretin (TTR), a recently identified protease, participates in the biology of high density lipoprotein and in the nervous system. In the present study, we determined whether TTR from a non-mammal Crocodylus porosus (crocTTR) has proteolytic activity and whether the N- and C-termini of the TTR polypeptide affect the proteolytic activity. The proteolytic activity of crocTTR and three chimeric crocTTRs: xenoN/crocTTR (crocTTR in which the $\mathrm{N}$-terminal sequence was replaced with that of Xenopus laevis TTR), pigC/crocTTR (crocTTR in which the C-terminal sequence was replaced with that of Sus scrofa TTR), and xenoN/pigC/crocTTR (crocTTR in which the N-and C-terminal sequences were replaced with that of $X$. laevis TTR and $S$. scrofa TTR, respectively) were studied and compared. Using either casein or apoAI as a substrate, crocTTR had a lower proteolytic activity than human TTR. Replacing the C-terminal sequence of crocTTR increased the activity (casein: $1008 \pm 36 \mathrm{pmol} / \mathrm{min}$; apoAI: $231 \pm 43 \mathrm{pmol} / \mathrm{min}$ ), whereas replacing the N-terminal sequence decreased the activity (casein: $299 \pm 26 \mathrm{pmol} / \mathrm{min}$; apoAI: $31.5 \pm 2.0 \mathrm{pmol} / \mathrm{min}$ ). The activity of xenoN/pigC/crocTTR (casein: $502 \pm 11 \mathrm{pmol} / \mathrm{min}$; apoAI: $371 \pm 23 \mathrm{pmol} / \mathrm{min}$ ) was higher than those of crocTTR or xenoN/crocTTR, but similar to that of pigC/crocTTR. These results are the first to show the proteolytic activity of reptile TTR, and that the activity is changed when the N- and/or C-terminal amino acid sequences of the TTR subunit are changed.
\end{abstract}

KEYWORDS: apolipoprotein A-I, evolutionary structure and function, FITC-casein, Xenopus laevis

\section{INTRODUCTION}

Transthyretin (TTR) is a thyroid hormone (TH) distributor protein found in the plasma and cerebrospinal fluid of most vertebrates. It is a tetramer consisting of four identical subunits in which the amino acid sequence has been highly conserved. The predominant changes in the TTRs from birds, reptiles, amphibians, and fishes are in the N-terminal region which is longer and more hydrophobic in the sequences compared to that in TTRs from mammals $\mathrm{s}^{1,2}$. In addition, variations in the length of the C-terminal region of TTRs from $\mathrm{pig}^{3}$ and microbats ${ }^{4,5}$ compared with that from human have also been detected. The changes in length and hydrophobicity were demonstrated to affect the binding affinities of TTR for THs and retinol binding protein (RBP) ${ }^{6,7}$.

Recently, the proteolytic activity of TTR was discovered and its participation in the biology of the nervous system and high density lipoprotein (HDL) was demonstrated ${ }^{8,9}$. The cleavages of its particular substrates including amyloid $\beta$ peptide $(\mathrm{A} \beta$ ) and amidated neuropeptide Y (NPY) can lead to the protection of amyloidogenesis and the enhancement of nerve cell regeneration ${ }^{10-12}$. On the other hand, the TTR cleavage on apolipoprotein AI (apoAI) promotes cholesterol efflux and increases amyloidogenicity of apoAI ${ }^{13}$. Based on the cleavage of apoAI, TTR was classified as a cryptic serine protease that specifically cleaves the substrate after Phe225 at the optimal $\mathrm{pH}$ of $7^{8}$. Subsequently, it was demonstrated to be a metallopeptidase with an inducible $\mathrm{Zn}^{2+}$-binding site ${ }^{14}$; in addition, the amino acid residues His88, His90, and Glu92 in an HXHXE motif serve as the complex formation site for the ligand. Since the sequence of HXHXE motif is conserved only in the TTRs from human and 
some non-human primates, the proteolytic activity of TTR has been claimed to be a recent evolutionary event ${ }^{14}$.

The phylogenetic trees which are based on the TTR primary structures and the morphological structure of vertebrates indicated that the structure of TTR evolved under functional pressures and provided an advantage for selection. For example, in the most well known function of TTR as a distributor for THs, mammalian TTRs have the binding preference opposite to the other vertebrate TTRs. While mammalian TTRs bind T4 better than T3, the other vertebrate TTRs bind T3 better than T4 ${ }^{15-17}$. The preference of the binding with $\mathrm{T} 4$ in mammalian TTRs correlates with the increase of the relative size of cerebral cortex, the requirement for $\mathrm{T} 4$ movement from blood to the brain, and the homoeostasis of THs in the choroid plexus in particular for proper growth and differentiation of the central nervous system of the animals ${ }^{18}$. The change of the binding favour of $\mathrm{TH}$ also reflexes with less effect of T4 on the metabolism of mammals. In addition, the concentrations of the total and free $\mathrm{T} 4$, and the tissue specific and the timing of the expression of the TTR gene in mammals differ from that of other vertebrates $^{19-21}$. The stepwise conversion of exon 2 into intron 1 sequences in the TTR gene constitutes the underlying mechanism of the structural change and thus favours TH binding ${ }^{1,2}$. We hypothesized that the activity of TTR also exists and has important roles in other classes of vertebrates, and the selection pressures that led to the structural changes of TTR during evolution would have influenced on this function. There is thus a need to characterize and compare the proteolytic activities between the TTRs from mammals and other classes of vertebrates, in addition to the evolutionary timing of these activities.

The major sites of TTR synthesis in most vertebrates are the liver and the choroid plexus of the brain. TTR synthesized in the choroid plexus is exclusively secreted into the CSF and functions within the brain ${ }^{22,23}$, whereas that synthesized in the liver is secreted and functions in the blood circulation. The evolutionary timing of TTR expression in each tissue is different among vertebrates. The synthesis in the liver first appeared earlier, during the development stages of fish and amphibians ${ }^{16}$, whereas the synthesis in the choroid plexus first appeared in the stem-reptiles ${ }^{24}$. In reptiles, the expression of TTR gene in the liver was observed during the development of juvenile but only for a short period of life; whereas the expression in the brain was detected throughout the rest of the animal's entire life ${ }^{21}$. These findings indicate the impact and specific requirement for TTR in the brain of the animals. Since the proteolytic activity of TTR has been reported only in human but not at all in other classes of vertebrates, TTR from Crocodylus porosus was thus chosen as a non-mammalian TTR to determine its proteolytic activity.

According to the X-ray crystal structure of human TTR, the $\mathrm{N}$ - and C-termini are flexible unstructured segments that protrude near the entrance to a central channel in which $\mathrm{TH}$ binds ${ }^{25}$. In addition, changes in the length and hydropathy of the terminal regions of TTR polypeptide have been clearly demonstrated to affect the properties that are related to the functions of TTR, i.e., the affinities of the bindings to THs and $\mathrm{RBP}^{7}$. The question arose whether the $\mathrm{N}$ - and C-terminal sequences affect the proteolytic activity of $C$. porosus TTR. Among the mature TTR polypeptides that have been identified, crocTTR has a length and hydropathy of the N-terminal sequence in between that of eutherian TTRs and Xenopus laevis TTR $^{2}$. To ascertain the effects of the longer and more hydrophobic amino acid sequences of the $\mathrm{N}$ - and C-termini on the proteolytic activity of the reptile TTR, three recombinant chimeric crocTTRs including xenoN/crocTTR (crocTTR in which the N-terminal sequence was replaced by the $\mathrm{N}$-terminal sequence of $X$. laevis TTR), pigC/crocTTR (crocTTR in which the C-terminal sequence was replaced by the C-terminal sequence of Sus scrofa TTR), and xenoN/pigC/crocTTR (crocTTR in which $\mathrm{N}$ - and C-terminal sequences were replaced by the $\mathrm{N}$ - and C-terminal sequences of $X$. laevis TTR and $S$. scrofa TTR, respectively) were synthesized, and their proteolytic activities were determined and compared.

The data presented here reveal that the proteolytic activity of TTR was not a recent evolutionary event but evolved since the early evolution of vertebrates; in addition, the effects of the $\mathrm{N}$ - and $\mathrm{C}$-terminal sequences on the proteolytic activity of the reptile TTR were demonstrated.

\section{MATERIALS AND METHODS}

\section{Reagents and chemicals}

Oligonucleotide primers were synthesized by Operon (Eurofins MWG Operon, Huntsville, AL, USA). PCR and plasmid purification kits were purchased from GibcoBRL (Long Island, NY, USA) and Qiagen (Hilden, Germany), and DNA ligase was from New England Biolabs (Ipswich, MA, USA). Taq DNA 
Table 1 Specific oligonucleotide primers used to generate cDNAs for chimeric crocTTRs. PCR was performed with the primers listed in three steps to generate cDNAs for pigC/crocTTR and xenoN/pigC/crocTTR. The nucleotide sequence of $C$. porosus TTR in the primers is underlined, that of $S$. scrofa TTR is double underlined, and that of $X$. laevis TTR is in bold.

\begin{tabular}{|c|c|c|c|}
\hline Species of TTR cDNA encoded & PCR step & Primer $5^{\prime} \rightarrow 3^{\prime}$ & Sense \\
\hline \multirow[t]{3}{*}{ pigC/crocTTR } & 1 & $\begin{array}{l}\text { TCTCTCGAGAAAAGAGCCCCACTGGTTTCCC } \\
\text { TGGGGCTGCTGACGAGGGCTGTG }\end{array}$ & $\begin{array}{l}\text { sense } \\
\text { antisense }\end{array}$ \\
\hline & 2 & $\begin{array}{l}\text { TCTCTCGAGAAAAGAGCCCCACTGGTTTCCC } \\
\text { AGCTCCCTCCTTGGGGCTGCTGAC }\end{array}$ & $\begin{array}{l}\text { sense } \\
\text { antisense }\end{array}$ \\
\hline & 3 & $\begin{array}{l}\text { TCTCTCGAGAAAAGAGCCCCACTGGTTTCCC } \\
\text { ACGGAATTCTCAAAGAGCTCCCTCCTTGGG }\end{array}$ & $\begin{array}{l}\text { sense } \\
\text { antisense }\end{array}$ \\
\hline \multirow[t]{3}{*}{ xenoN/pigC/crocTTR } & 1 & $\begin{array}{l}\text { TCCCATGGAGAAGCCGATTCC } \\
\text { TGGGGCTGCTGACGAGGGCTGTG }\end{array}$ & $\begin{array}{l}\text { sense } \\
\text { antisense }\end{array}$ \\
\hline & 2 & $\begin{array}{l}\text { ACCGGGACATGCTTCCCATGG } \\
\text { AGCTCCCTCCTTGGGGCTGCTGAC } \\
\end{array}$ & $\begin{array}{l}\text { sense } \\
\text { antisense }\end{array}$ \\
\hline & 3 & $\begin{array}{l}\text { CTCGAGAAAAGAGCACCACCGGGACATGC } \\
\text { ACGGAATTCTCAAAGAGCTCCCTCCTTGGG }\end{array}$ & $\begin{array}{l}\text { sense } \\
\text { antisense }\end{array}$ \\
\hline
\end{tabular}

polymerase was a product of Invitrogen (Carlsbad, CA, USA). Horseradish peroxidase conjugated anti rabbit IgG and the ECL kit were from Calbiochem (San Diego, CA, USA) and Amersham Bioscience (Chalfont St Giles, UK), respectively. Human RBP purified from urine was from RDI (Fitzgerald, MA, USA). Cibacron blue 3GA and fluorescein isothiocyanate (FITC)-casein were products of Sigma (St Louis, MO, USA). Human apoAI was from Fitzgerald (Acton, MA, USA). All other chemicals used were of analytical grade.

\section{Construction, cloning, and expression of TTR cDNAs}

Two new chimeric TTR cDNAs were constructed and amplified by PCR. These included pigC/crocTTR cDNA which consisted of amino acid residues 1114 of crocTTR and residues 115-130 of $S$. scrofa TTR, and xenoN/pigC/crocTTR cDNA which consisted of amino acid residues 1-9 of $X$. leavis TTR, residues 10-113 of crocTTR, and residues 114-130 of $S$. scrofa TTR. PCR was performed using specific primers (Table 1). The nucleotide sequences of the cDNAs were confirmed by sequencing before they were ligated into a Pichia expression vector, pPIC9. The recombinant vectors were transformed into $P$. pastoris and the transformant clones were selected as described previously ${ }^{6,7}$.

\section{Production and purification of recombinant TTRs}

The induction of Pichia clones to produce all the studied recombinant and chimeric TTRs was per- formed as described previously ${ }^{6,7}$. In brief, the Pichia clones of crocTTR ${ }^{17}$, xenoN/crocTTR ${ }^{17}$ and the two new chimeric crocTTRs were induced with $0.5 \%$ methanol for 3 days at $28^{\circ} \mathrm{C}$ in buffered complex glycerol or methanol medium (BMGY/BMMY). TTRs were purified from the culture supernatant by preparative discontinuous native-PAGE using BioRad prep cell model 491 (12\% acrylamide resolving gel and $4 \%$ stacking gel).

\section{Purification of human TTR from plasma}

Human plasma was applied onto a Cibacron blue column that was pre-equilibrated with $50 \mathrm{mM}$ phosphate buffer, $\mathrm{pH}$ 7.4. Thereafter, the column was washed and unbound proteins were collected for further purification by preparative discontinuous native-PAGE using the same conditions as described for the recombinant TTRs.

\section{Analysis of the physicochemical properties of TTRs}

The subunit masses of the chimeric pigC/crocTTR and xenoN/pigC/crocTTR were determined by SDSPAGE (12\% resolving gel and 4\% stacking gel) in the presence of 2.5\% 2-mercaptoethanol and 2\% SDS, and followed by Coomassie blue staining. The sizes of the TTR subunits were obtained by comparing their relative mobilities with protein markers: phosphorylase b (94 kDa), bovine serum albumin (67 kDa), ovalbumin (43 kDa), carbonic anhydrase (30 kDa), soya bean trypsin inhibitor $(20.1 \mathrm{kDa})$, and $\alpha$-lactalbumin (14.4 kDa). Crossed-reactivity of the recombinant TTRs was determined by western 
blotting, followed by enhanced chemiluminescence (ECL) detection as previously described ${ }^{6,7}$. The primary and secondary antibodies used were anticrocTTR antibody raised in rabbit (dilution 1:2500) and horseradish peroxidase-conjugated anti-rabbit IgG antibody (dilution 1:10000), respectively. The electrophoretic mobilities of TTRs under native condition were determined by native-PAGE (10\% resolving gel and $4 \%$ stacking gel), and the molecular weights of the tetramers were determined by HPLC/gel-permeation chromatography using a Biosil-SEC 250 column $(300 \times 7.8 \mathrm{~mm})$ that was pre-equilibrated with $0.2 \mathrm{M}$ phosphate buffer, $\mathrm{pH}$ 6. The separation was carried out at a flow rate of $0.3 \mathrm{ml} / \mathrm{min}$. The standard proteins that were used to calibrate the column were aldolase $(158000 \mathrm{kDa})$, albumin $(67000 \mathrm{kDa})$, ovalbumin $(43000 \mathrm{kDa})$, and ribonuclease A (13700 kDa). The partition coefficient $\left(K_{\mathrm{av}}\right)$ values were plotted against log of the molecular weight of the standard proteins to generate the calibration curve. $K_{\mathrm{av}}$ was calculated using the formula $K_{\mathrm{av}}=\left(V_{\mathrm{e}}-V_{\mathrm{o}}\right) /\left(V_{\mathrm{t}}-V_{\mathrm{o}}\right)$ where $V_{\mathrm{e}}$ is the elution volume of the sample, $V_{\mathrm{o}}$ is the void volume and $V_{\mathrm{t}}$ is the total volume of the column.

\section{Hydropathy plots of TTRs}

The hydropathy profiles of recombinant crocTTR and chimeric TTRs were generated by the method of Kyte and Doolittle ${ }^{26}$, using a scanning window of seven amino acid residues. Differences in the hydropathic profiles of the chimeric TTRs compared with crocTTR were observed only at the $\mathrm{N}$ - and C-terminal regions (Fig. 1). The N-terminal sequences of xenoN/crocTTR and xenoN/pigC/ crocTTR were less hydrophobic than that of pigC/ crocTTR or crocTTR, whereas the C-terminal sequences of pigC/crocTTR and xenoN/pigC/crocTTR were slightly less hydrophobic than those of the xenoN/crocTTR and crocTTR.

\section{Determination of the binding to human RBP}

The bindings to human RBP of human TTR, crocTTR and chimeric TTRs were determined as described previously ${ }^{7}$. Purified TTR $(0.5 \mu \mathrm{M})$ was incubated with purified human RBP at TTR:RBP molar ratios of $1: 2,1: 4$, and $1: 8$. The reaction mixture was incubated at $4{ }^{\circ} \mathrm{C}$ for $1 \mathrm{~h}$. The binding between TTR and RBP was analysed by native-PAGE ( $8 \%$ resolving and 4\% stacking) prior to the protein transfer onto a PVDF membrane. Bound and unbound TTRs were detected by western analysis followed by ECL using anti-crocTTR antibody (dilution 1:500)

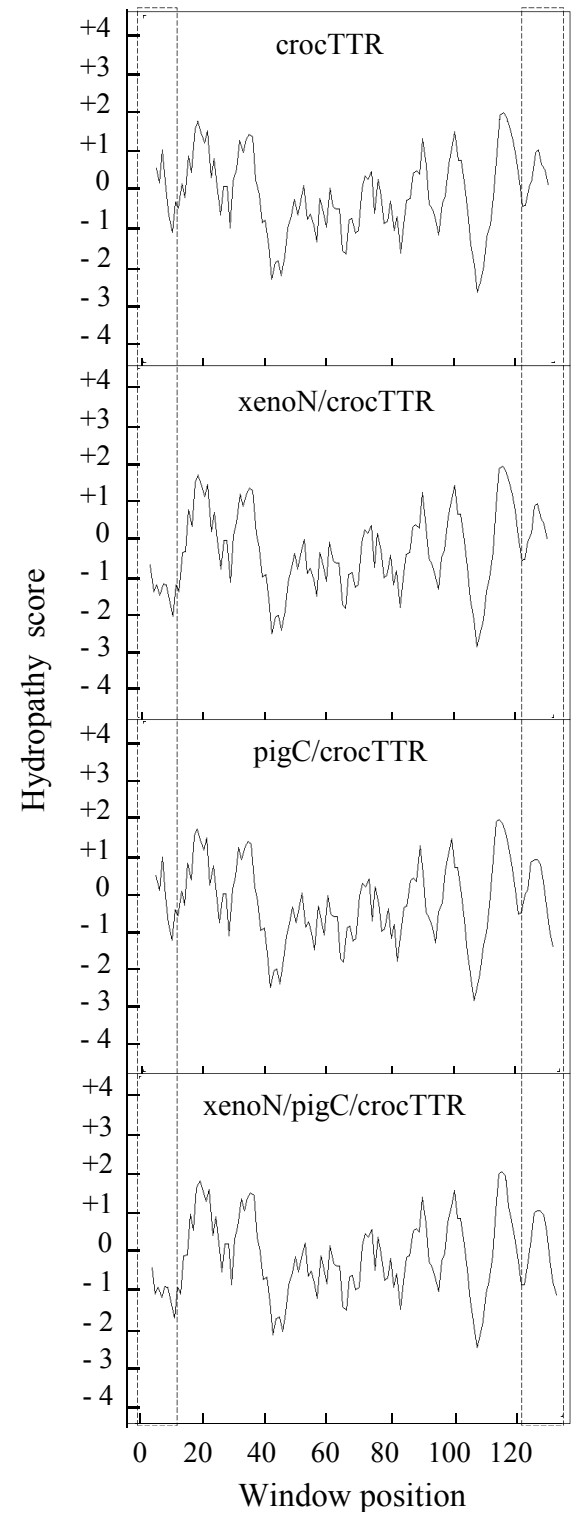

Fig. 1 Comparison of the hydrophobicity plots of the crocTTR and chimeric TTRs. A scanning window of 7 amino acid residues was used. Broken blocks indicate the $\mathrm{N}$ - and $\mathrm{C}$-terminal regions at which the amino acid sequences were changed.

and horseradish peroxidase-conjugated anti-rabbit IgG antibody (dilution 1:1000) as the primary and secondary antibodies, respectively.

\section{Thermal stability assay}

To determine the effect of temperature on the proteolytic activity, aliquots of TTR in $50 \mathrm{mM}$ Tris- $\mathrm{HCl}$, $\mathrm{pH} 7.4$, were incubated at a constant temperature (from $0^{\circ} \mathrm{C}$ to $70^{\circ} \mathrm{C}$ ) for $30 \mathrm{~min}$, and then the solu- 
tion was immediately cooled in ice water prior to the assay for the activity at $37^{\circ} \mathrm{C}$ using FITC-casein as substrate. The relative specific activities to that at $0^{\circ} \mathrm{C}$ were calculated. The reaction of the FITCcasein alone was included to correct for possible thermal effects on the substrate.

\section{Proteolytic activity assays}

The concentration of purified TTR was determined by Bradford protein assay ${ }^{27}$. The caseinolytic activities of human TTR, recombinant crocTTR and chimeric TTRs were analysed as described by Twining ${ }^{28}$. Aseptic technique was performed to avoid possible contamination with microorganisms. A reaction mixture $(10 \mu \mathrm{l})$ in $50 \mathrm{mM}$ Tris- $\mathrm{HCl}, \mathrm{pH} 7.4$, containing $1 \mu \mathrm{g}(0.01 \mathrm{nmol})$ of purified TTR, $0.2 \%$ FITC-casein, $0.2 \mathrm{M} \mathrm{NaCl}$ and $5 \mathrm{mM} \mathrm{CaCl}{ }_{2}$, was incubated for a constant period $(0-24 \mathrm{~h})$ in the dark. The reaction was terminated with $0.6 \mathrm{~N}$ trichloroacetic acid. The insoluble protein pellet was removed by centrifugation at $10000 \mathrm{rpm}$ for $20 \mathrm{~min}$, at $4^{\circ} \mathrm{C}$. An aliquot of the clear supernatant was diluted with $500 \mathrm{mM}$ Tris- $\mathrm{HCl}, \mathrm{pH} 8.5$, before fluorescence intensity was measured by using a fluorospectrometer (Shimazu) at an excitation and emission wavelengths of $490 \mathrm{~nm}$ and $525 \mathrm{~nm}$, respectively. To correct for a possible thermal degradation of the FITC-casein, the reaction mixture of FITC-casein alone was included. Quinine hydrochloride was used as a fluorescence standard. The initial reaction rate was calculated from the increased free FITC. For each types of TTR, three independent experiments using different preparations of TTR were conducted, and the enzymatic assay of each experiment was performed in triplicate. The results are presented as activity of TTR (expressed in $\mathrm{pmol} / \mathrm{min}$ ).

For the assay of the cleavage of apoAI, $1 \mu \mathrm{g}$ $(0.01 \mathrm{nmol})$ of purified TTR was incubated with $1 \mu \mathrm{g}(0.03 \mathrm{nmol})$ of apoAI at $37^{\circ} \mathrm{C}$ in $50 \mathrm{mM}$ Tris, $\mathrm{pH}$ 7.5. The reaction was terminated by quickly freezing the mixture in liquid nitrogen. Thereafter, it was analysed by SDS-PAGE on a $20 \%$ polyacrylamide gel at $4{ }^{\circ} \mathrm{C}$ followed by Coomassie blue R250 staining. Intensity of the cleaved apoAI fragment was quantified by a gel documentation system (BioSpectrum Imagine System, UVP) with VISIONWORKS LS. For each type of TTR, three independent experiments using different TTR preparations were conducted, and the enzymatic assay for each experiment was performed in triplicate. The initial reaction rate of TTR was determined from the increase of cleaved apoAI (compared to the total apoAI at the start point, $t=0$ ). The results were presented as activity of TTR expressed in $\mathrm{pmol} / \mathrm{min}$.

\section{Statistical analysis}

Unless otherwise specified, data are presented as the mean value \pm SEM. Results were compared using one way ANOVA, and a post-hoc test with student $t$-test to compare between two groups, $p$ values of less than $0.05(p<0.05)$ were considered to be significant.

\section{RESULTS}

\section{Synthesis and characterization of recombinant TTRs}

The characteristics of pigC/crocTTR and xenoN/ pigC/crocTTR indicated that they were synthesized and extracellularly secreted as functioning molecules. The subunit mass of purified pigC/ crocTTR was $14.7 \mathrm{kDa}$ (Fig. 2a) and was similar to that of crocTTR, whereas the subunit mass of xenoN/pigC/crocTTR was $15.6 \mathrm{kDa}$ which was slightly larger than that of crocTTR but similar to that of xenoN/crocTTR ${ }^{17}$. The relative masses of the tetrameric molecules determined by HPLC were $63 \mathrm{kDa}$ for pigC/crocTTR and $69 \mathrm{kDa}$ for xenoN/ pigC/crocTTR (Fig. 2b). These relative masses were four-fold higher than that of the respective subunits, indicating that Pichia synthesized these TTRs as tetrameric molecules and no glycosylation in particular occurred. Both pigC/crocTTR and xenoN/ pigC/crocTTR possessed other characteristics similar to those previously produced in $P$. pastoris, i.e., had cross-reactivity with a specific antibody raised against crocTTR (Fig. 2a) and the electrophoretic mobility under native conditions at $\mathrm{pH} 8.8$ was faster than that of serum albumin, but similar to that of human TTR purified from plasma (Fig. 2c). In addition, both TTRs were able to bind human RBP (Fig. 3).

\section{Temperature stability of TTRs}

The thermal degradation of FITC-casein was not significantly observed within the examined temperature range (data not shown). In comparison, the caseinolytic activities of human TTR and crocTTR were not significantly changed when the temperature was increased from $0^{\circ} \mathrm{C}$ to $40^{\circ} \mathrm{C}$ (Fig. 4). The activity decreased when the temperature was higher than $40^{\circ} \mathrm{C}$. In comparison to human TTR, a more rapid loss of the activity was observed for crocTTR. At $50{ }^{\circ} \mathrm{C}$, the activity of crocTTR was less than $50 \%$ whereas that of human TTR was $\sim 60 \%$ (Fig. 4). 

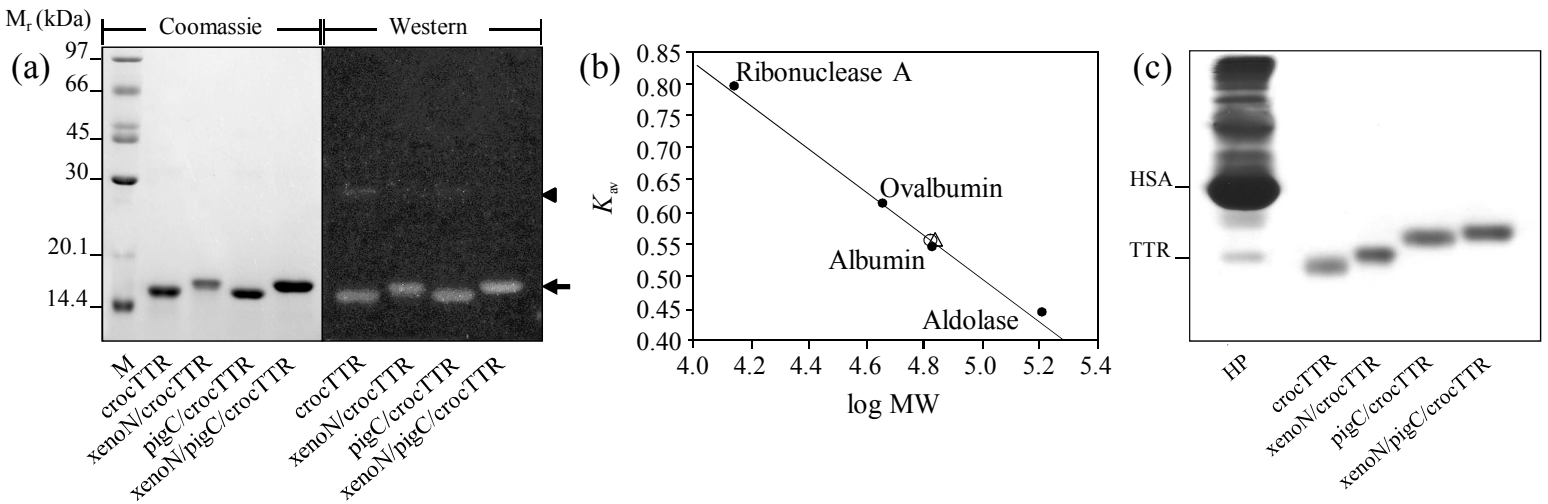

Fig. 2 Characterization of recombinant TTRs. (a) Determination of the TTR subunit masses by SDS-PAGE followed by Coomassie blue staining (Coomassie), and western analysis (Western) with antiserum against crocTTR, followed by detection of ECL. Sizes of the TTR subunits were obtained by comparing their relative mobilities with protein markers (M). The positions of the TTR monomer and dimer are indicated by an arrow and arrow head, respectively. (b) The calibration curve of the Biosil-SEC 250 column. Opened circular and opened triangles show the positions corresponding to the $K_{\mathrm{av}}$ of pigC/crocTTR and xenoN/pigC/crocTTR, respectively. (c) The electrophoretic mobilities of TTRs on the native-polyacrylamide gel. Human plasma (HP) was loaded to show the position of human TTR (TTR) and serum albumin (HSA).

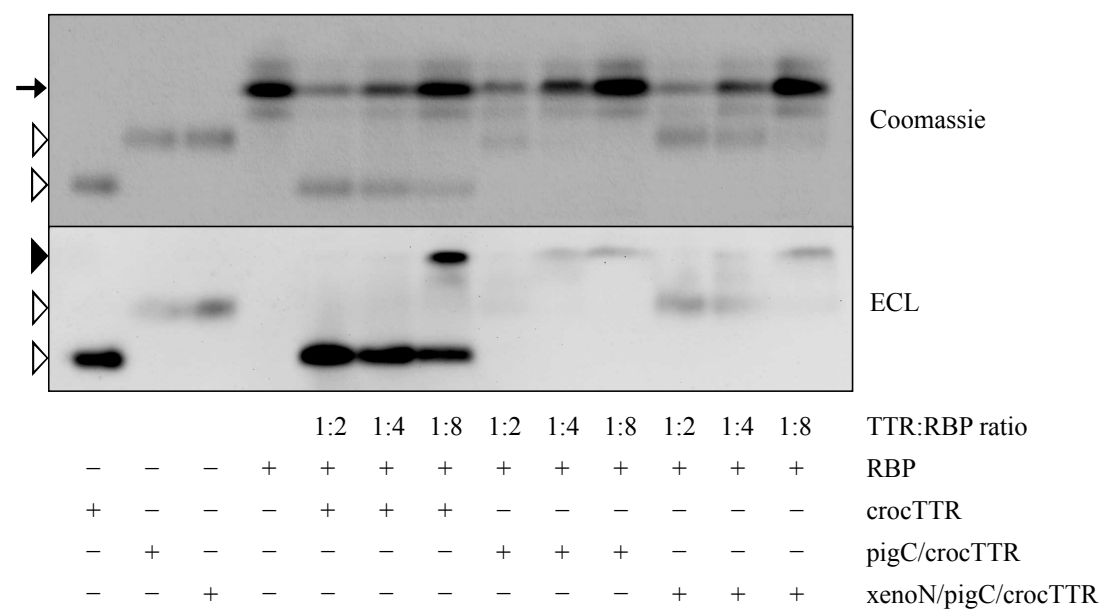

Fig. 3 Analysis of the binding between TTRs and human RBP. An aliquot $(0.5 \mu \mathrm{M})$ of purified TTR was incubated with human RBP at the TTR:RBP molar ratios of $1: 2,1: 4$ and $1: 8$, at $4^{\circ} \mathrm{C}$ for $1 \mathrm{~h}$. Then the reaction mixture was analysed by native-PAGE (10\% resolving and 4\% stacking gels). Proteins were identified by Coomassie blue staining (Coomassie). Bound and free TTRs were identified by western blotting followed by ECL (ECL), using antibody specific to the crocTTR as the primary antibody. The TTRs and RBP alone were added as controls. The arrow indicates free RBP. The open and closed arrowheads indicate free and bound TTRs, respectively.

The proteolytic activities using a casein substrate of crocTTR and human TTR were almost totally destroyed when the temperature was increased to $70^{\circ} \mathrm{C}$.

\section{Caseinolytic activities of TTRs}

The ability of recombinant crocTTR to cleave FITCcasein was about one third of that of human TTR (mean \pm SEM: $199 \pm 11$ versus $666 \pm 19 \mathrm{pmol} / \mathrm{min}$ ).
The difference of the caseinolytic activity between crocTTR and chimeric TTRs indicated that the Nand C-terminal regions in particular influenced the activity (Fig. 5). All the chimeric crocTTRs had higher caseinolytic activity than the crocTTR. The activities of xenoN/crocTTR $(299 \pm 26 \mathrm{pmol} / \mathrm{min})$, $\mathrm{pigC} / \mathrm{crocTTR}(1008 \pm 36 \mathrm{pmol} / \mathrm{min})$, and xenoN/ $\mathrm{pigC} / \mathrm{crocTTR}(502 \pm 11 \mathrm{pmol} / \mathrm{min})$ were $1.5-$, 5-, and 2.5-fold higher than that of crocTTR, respec- 


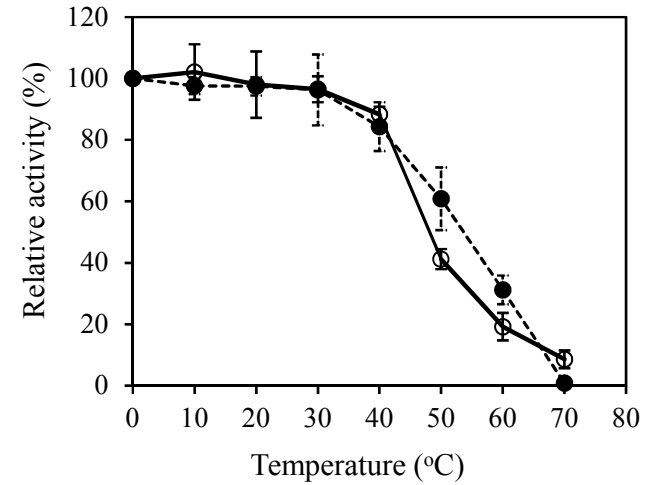

Fig. 4 Thermal stability profiles of human TTR and crocTTR. The solutions of human TTR (closed circle) and crocTTR (open circle) were incubated in the absence of substrate at $0{ }^{\circ} \mathrm{C}$ to $70^{\circ} \mathrm{C}$. After the treatment, the proteolytic activity for FITC-casein at $37^{\circ} \mathrm{C}$ was performed. The relative specific activities to that at $0^{\circ} \mathrm{C}$ were calculated. Results were from triplicate assays and are presented as mean values; error bars represent standard deviations.

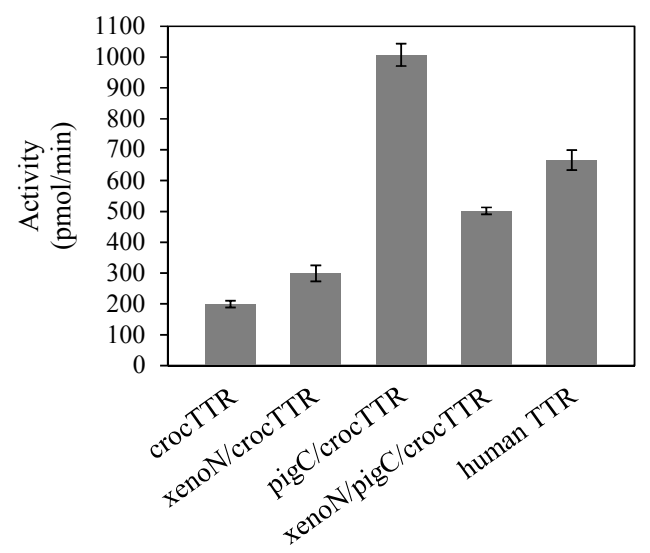

Fig. 5 Proteolytic activities of TTRs for FITC-casein. The reaction mixture contains purified TTR and $0.2 \%$ FITC-casein. The fluorescence intensity of the catalytic released FITC was measured at an excitation and emission wavelengths of 490 and $525 \mathrm{~nm}$, respectively. Quinine hydrochloride was used as a fluorescence standard. Results are presented as mean values; error bars represent the SEM. All mean values are significantly different at $p<0.05$.

tively. However, only pigC/crocTTR had a caseinolytic activity higher than that of human TTR.

\section{ApoAI cleavage by TTRs}

At $37^{\circ} \mathrm{C}$, a decrease of the intensity of the uncleaved apoAI band $(\sim 26 \mathrm{kDa})$ and the increase of intensity of the cleaved apoAI band over time was observed (a)
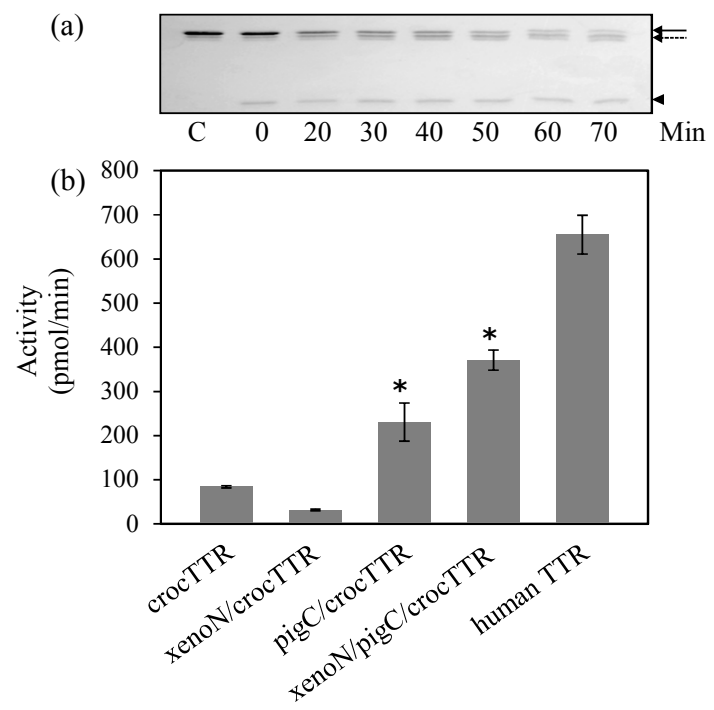

Fig. 6 The proteolytic activity of TTRs for apoAI. (a) The representative apoAI cleavage pattern as analysed by SDSPAGE. A mixture of purified TTR and apoAI was incubated at $37^{\circ} \mathrm{C}$ for various periods. Then the reaction was analysed on a $20 \%$ polyacrylamide gel. The gel was stained with Coomassie blue R-250. The arrow, uncleaved apoAI; the broken arrow, cleaved apoAI; the arrow head, the position of the TTR monomer. (b) The intensity of the cleaved apoAI was measured and analysed by a gel documentation system with VISIONWORKS LS. Results are presented as mean values; error bars represent SEM. Values marked with * were not significantly different at $p<0.05$.

in the reactions of apoAI with TTR (Fig. 6a). The cleaved apoAI fragments appeared as one to two bands of lower molecular weight than the uncleaved apoAI, as observed previously ${ }^{8}$.

Both the recombinant crocTTR and chimeric crocTTRs cleaved apoAI, and the TTR with longer and more hydrophilic C-terminal region showed higher activity of the apoAI cleavage (Fig. 6b). The apoAI cleavage activity of crocTTR (mean \pm SEM: $84.0 \pm 2.5 \mathrm{pmol} / \mathrm{min}$ ) was 8 -fold lower than that of human TTR ( $655 \pm 44 \mathrm{pmol} / \mathrm{min})$. In comparison to crocTTR, xenoN/crocTTR that contains a longer and more hydrophilic $\mathrm{N}$-terminal sequence had lower activity of the apoAI cleavage $(31.5 \pm 2.0 \mathrm{pmol} / \mathrm{min})$; however, the chimeric crocTTRs those contain longer and more hydrophilic C-terminal sequences, including pigC/crocTTR and xenoN/pigC/crocTTR, had higher the activity. The activities of pigC/ crocTTR and xenoN/pigC/crocTTR were $231 \pm 43$ and $371 \pm 23 \mathrm{pmol} / \mathrm{min}$, respectively. 


\section{DISCUSSION}

Because TTR is synthesized in the choroid plexus of C. porosus but only in relatively small amounts ${ }^{17}$, the recombinant crocTTR and chimeric TTRs were thus produced using the protein expression system of $P$ pastoris in order to obtain sufficient amounts of the protein for further analysis. The recombinant crocTTR and chimeric TTRs produced in this study had the physicochemical properties and the ability to bind with specific ligands as previously reported $^{6,7}$. This indicated that they were synthesized and secreted from the Pichia cells as functional tetramers.

TTR was firstly identified as a serine protease because its activity was inhibited by the inhibitors that are specific for serine and Kunitz proteases ${ }^{8,10}$; however, its catalytic triad was not identified ${ }^{8}$. Later, its catalytic activity was shown to be inducible by $\mathrm{Zn}^{2+}$, and consequently, TTR was claimed to be a metalloproteinase ${ }^{14}$. It seems likely that the catalytic mechanism of TTR is still not totally clear. In our experiments, casein and apoAI were chosen because casein is a well known universal substrate particular for serine proteases $8,28,29$ while apoAI is a specific natural substrate of $\mathrm{TTR}^{12}$. Although both substrates have the cleavage site(s) for TTR, the molecules have some different characteristics. According to the amino acid sequence, casein is expected to contain more than a single cleavage site for TTR, whereas, only a single site was reported on apoAI ${ }^{8}$. The structure of casein contains several types of subunits which vary primarily in molecular weight, the isoelectric point, and the level of phosphorylation, leading to several secondary structures including an $\alpha$-helix and a $\beta$-sheet of the molecule ${ }^{30}$. In contrast, the primary structure of apoAI is composed of several tandem repeats of 22 amino acids that form the amphipathic $\alpha$-helical secondary structure ${ }^{31}$. In comparison, apoAI has a much simpler structure than casein. Accordingly, the cleavage site of apoAI could be more specifically accessible to TTR. In consideration of the structure of TTR which is composed of four subunits, it could be conceivable that not only the specific affinity but also the accessibility of the substrate to the catalytic site are important factors that determine the catalytic rate of TTR. In addition, the studied TTRs contained different amino acid sequences particular in the $\mathrm{N}$ - and/or C-terminal regions. This could generate different new environments among the TTRs, that affected the accessibility of the substrate and stability of the interactions between TTR and the substrate (casein or apoAI). Taken together, the concerted effects of TTR and substrate structures, and the new environment generated by the changed amino acid sequences at the $\mathrm{N}$ - or $\mathrm{C}$-terminal region of TTRs could lead to differences in the catalytic activity of each type of TTR with different substrates as observed in our experimental results.

Based on the finding that the $\mathrm{Zn}^{2+}$-binding motif is important for the proteolytic activity of TTR and it is conserved only in the amino acid sequences of human and some non-human primate TTRs, the proteolytic activity of TTR is believed to be a recent evolutionary event ${ }^{14}$. Our present findings first showed that a non-mammalian TTR, i.e., C. porosus TTR (crocTTR) had proteolytic activity with a thermal stability comparable to that of human TTR (Fig. 4). Although the activity of crocTTR was lower than that of human TTR, its specific cleavage pattern for apoAI was similar to that of human TTR. In addition, the proteolytic activity of crocTTR could be altered by changing the length and hydropathy of the amino acid sequences at the $\mathrm{N}$ - and C-terminal regions. Together with the fact that apoAI is a natural substrate of $\mathrm{TTR}^{8}$, we propose that the proteolytic activity of TTR appeared early in the evolution of vertebrates, and thus its function in the brain probably first existed in the stem-reptiles.

The specific cleavage of apoAI by crocTTR and by human TTR was similar, and it was correlated with the fact that the primary structure of apoAI has been conserved among the vertebrate species ${ }^{32}$. Since apoAI of the vertebrates has at least three main functions, i.e., the association with lipids and cholesterol, binding to HDL binding receptors, and the activation of lecithin:cholesterol acyl transferase, the ability of $C$. porosus TTR to specifically cleave apoAI could indicate to a possible involvement of TTR on the regulation of apoAI for the same particular functions in the reptile.

In the polypeptide chain of crocTTR, the $\mathrm{Zn}^{2+}$. chelating sequence HXHXE that is present in human TTR does not exist. In crocTTR polypeptide, the presence of His 88 and Glu92 is the same as in the TTRs from human and other primates; however, the amino acid at position 90 of the reptile TTR is Tyr, but not His ${ }^{17}$. Lower proteolytic activity was observed in crocTTR compared with human TTR, therefore, it could support the importance of His90 for the $\mathrm{Zn}^{2+}$ binding and, thus the proteolytic activity of TTR. His and Tyr interact with $\mathrm{Zn}^{2+}$ with different affinities. In solution, His mostly interacts with $\mathrm{Zn}^{2+}$ via a coordinate interaction in which the lone electron pair of the basic $\mathrm{N}$ in the imidazole 
ring acts as a coordinate ligand for the metallic cations ${ }^{33}$, whereas, the Tyr and $\mathrm{Zn}^{2+}$ complex was stabilized by a cation- $\pi$ interaction, and a noncovalent binding between an aromatic $\pi$ system and a metallic ion ${ }^{34}$. A comparative determination of the binding affinities between crocTTR and human TTR with $\mathrm{Zn}^{2+}$ is therefore required to confirm the importance of the His residue in the HXHXE motif. $\mathrm{Zn}^{2+}$ at a high concentration could bind to the binding site near to the active site of TTR and inhibit the proteolytic activity of the protein ${ }^{14}$. Although the amount of metal ions, particularly $\mathrm{Zn}^{2+}$, in our preparation of the recombinant crocTTR, was not determined, the experiment with additional $\mathrm{Zn}^{2+}$ showed no significant change in the activity (data not shown). This could indicate that $\mathrm{Zn}^{2+}$ was a contaminant in the preparation. The possibility of lowering the activity of crocTTR by contaminating metal ions, therefore, cannot be excluded.

C. porosus TTR and its chimeric TTRs have a cysteine at position 10. Post-translational modification of cysteine such as glutathionylation in particular has been reported in human $\mathrm{TTR}^{35}$. In addition, hyperglycosylation was also reported for the recombinant protein produced by $P$. pastoris ${ }^{36}$. These possible modifications could affect the activity of the TTR. According to our most recent knowledge, however, cysteine at position 10 or at other positions on the TTR polypeptide is not involved in the catalytic site of TTR ${ }^{14}$. Although glutathionylation and other modifications of the cysteine at position 10 on the TTR polypeptide were reported, the modifications usually resulted in a lower stability of the TTR tetramer and led to the formation of TTR-related amyloid fibril ${ }^{37}$ with a lower binding ability of TTR with $\mathrm{T}^{38}$. Thus a possible modification on cysteine at position 10 is unlikely, but not totally be excluded, to affect the proteolytic activity of the reptile TTR that was observed in this study.

The comparison of the amino acid sequences of vertebrate TTRs showed that the amino acid residues involved in the binding of TTR to THs remained unchanged during evolution. However, changes in the amino acid sequence during evolution are concentrated in the $\mathrm{N}$-terminal region of the TTR subunit, and produce variations in the length and hydropathy of amino acid sequence in the C-terminal region of TTR subunits as are also observed in some animal species including pig $^{3}$ and bats ${ }^{4,5}$. In a previous work, we demonstrated that the unstructured $\mathrm{N}$-terminal region of TTR had effects on the affinity of the binding between TTR and $\mathrm{TH}^{6}$, and it also affected the binding of RBP to the binding site at the C-terminal region of $\mathrm{TTR}^{7}$. Hereto, the $\mathrm{N}$ - and C-terminal sequences of TTR also had effects on its proteolytic activity. The proteolytic activity decreased when the N-terminal sequence of the subunit was longer and more hydrophilic (e.g., xenoN/crocTTR), but increased when the C-terminal sequence was longer and more hydrophilic (e.g., $\mathrm{pigC} / \mathrm{crocTTR}$ ). It is of interest that, making both the $\mathrm{N}$ - and $\mathrm{C}$-termini of crocTTR to be longer and more hydrophilic (e.g., xenoN/pigC/crocTTR) resulted in a TTR with comparable proteolytic activity to that in which only the C-terminus was changed (e.g., pigC/crocTTR). In human TTR, the binding of $\mathrm{Zn}^{2+}$ to the His88, His90 and Glu92 motif is particularly important for the apoAI cleavage of $\mathrm{TTR}^{14}$. This motif is located in the C-terminal region that flanks the region involved in the recognition of $\mathrm{RBP}^{39,40}$. In a metalloproteinase, bound $\mathrm{Zn}^{2+}$ is responsible for catalysing the peptide hydrolysis, and the binding of the substrate to bound $\mathrm{Zn}^{2+}$ is an initial step of the reaction. Because none of the amino acid residues involved in the catalytic activity of TTR for apoAI are within the first 10 amino acid residues from the $\mathrm{N}$ - or $\mathrm{C}$-terminus of the TTR subunit, changes in the length and hydropathy of the polypeptide end-terminal regions would be expected to generate a new environment that interfered or facilitated the access of $\mathrm{Zn}^{2+}$ to its binding motif and/or of the apoAI to the bound $\mathrm{Zn}^{2+}$.

\section{CONCLUSIONS}

In summary, we determined the proteolytic activity of TTR from C. porosus and showed that the reptile TTR also had proteolytic activity for apoAI. Our data therefore first revealed the existence of the proteolytic activity in non-mammalian TTR. Consequently, the existence of the proteolytic activity of TTR since early in the evolution of vertebrates was suggested. Our obtained results showed that the proteolytic activity of crocTTR was changed when the amino acid sequences of its polypeptide terminal ends were changed, and together with our previous findings of the effects of the $\mathrm{N}$-terminal sequence of TTR on its binding affinities for $\mathrm{TH}^{6}$ and $\mathrm{RBP}^{7}$, we propose that both the $\mathrm{N}$ - and C-terminal regions have effects on the activity and, as a consequence, the function that is related to this property of TTR.

Acknowledgements: This work was supported by Prince of Songkla University and Rajanukul Institute, Thailand. Ladda Leelawatwattana received financial support from Thailand Research Fund, Office of the Higher Education Commission and Prince of Songkla 
University under contract number MRG5380196. We wish to express our thanks to Dr J Monk and Dr Brian Hodgson for assistance in editing the manuscript.

\section{REFERENCES}

1. Aldred AR, Prapunpoj P, Schreiber G (1997) Evolution of shorter and more hydrophilic transthyretin N-termini by stepwise conversion of exon 2 into intron 1 sequences (shifting the $3^{\prime}$ splice site of intron 1). Eur J Biochem 246, 401-9.

2. Prapunpoj P, Leelawatwattana L (2009) Evolutionary changes to transthyretin: structure-function relationships. FEBS J 276, 5330-41.

3. Duan W, Richardson SJ, Köhrle J, Chang L, Southwell BR, Harms PJ, Brack CM, Pettersson TM, Schreiber G (1995) Binding of thyroxine to pig transthyretin, its cDNA structure, and other properties. Eur J Biochem 230, 977-86.

4. Seim I, Fang X, Xiong Z, Lobanov AV, Huang Z, Ma S, Feng Y, Turanov AA, et al (2013) Genome analysis reveals insights into physiology and longevity of the Brandt's bat Myotis brandtii. Nat Comm 4, 2212.

5. Zhang G, Cowled C, Shi Z, Huang Z, Bishop-Lilly KA, Fang X, Wynne JW, Xiong Z, et al (2013) Comparative analysis of bat genomes provides insight into the evolution of flight and immunity. Science 339, 456-60.

6. Prapunpoj P, Leelawatwatana L, Schreiber G, Richardson SJ (2006) Change in structure of the $\mathrm{N}$-terminal region of transthyretin produces change in affinity of transthyretin to T4 and T3. FEBS J 273, 4013-23.

7. Leelawatwattana L, Praphanphoj V, Prapunpoj P (2011) Effect of the N-terminal sequence on the binding affinity of transthyretin for human retinolbinding protein. FEBS $J$ 278, 3337-47.

8. Liz MA, Faro CJ, Saraiva MJ, Sousa MM (2004) Transthyretin, a new cryptic protease. $J$ Biol Chem 279, 21431-8.

9. Liz MA, Mar FM, Franquinho F, Sousa MM (2010) Aboard transthyretin: from transport to cleavage. IUBMB Life 62, 429-35.

10. Costa R, Ferreira-da-Silva F, Saraiva MJ, Cardoso I (2008) Transthyretin protects against A-Beta peptide toxicity by proteolytic cleavage of the peptide: a mechanism sensitive to the Kunitz protease inhibitor. PLOS ONE 3, e2899.

11. Costa R, Gonçalves A, Saraiva MJ, Cardoso I (2008) Transthyretin binding to A-Beta peptide: impact on A-Beta fibrillogenesis and toxicity. FEBS Lett 582, 936-42.

12. Liz MA, Fleming CE, Nunes AF, Almeida MR, Mar FM, Choe Y, Craik CS, Powers JC, et al (2009) Substrate specificity of transthyretin: identification of natural substrates in the nervous system. Biochem $J 419$, 467-74.
13. Liz MA, Gomes CM, Saraiva MJ, Sousa MM (2007) ApoA-I cleaved by transthyretin has reduced ability to promote cholesterol efflux and increased amyloidogenicity. J Lipid Res 48, 2385-95.

14. Liz MA, Leite SC, Juliano L, Saraiva MJ, Damas AM, Bur D, Sousa MM (2012) Transthyretin is a metallopeptidase with an inducible active site. Biochem $J$ 443, 769-78.

15. Chang L, Munro SLA, Richardson SJ, Schreiber G (1999) Evolution of thyroid hormone binding by transthyretin in birds and mammals. Eur $J$ Biochem 259, 534-42.

16. Prapunpoj P, Yamauchi K, Nishiyama N, Richardson SJ, Schreiber G (2000) Evolution of structure, ontogeny of gene expression, and function of Xenopus laevis transthyretin. Am J Physiol Regul Integr Comp Physiol 279, R2026-41.

17. Prapunpoj P, Richardson S, Schreiber G (2002) Crocodile transthyretin: structure, function, and evolution. Am J Physiol Regul Integr Comp Physiol 283, R885-96.

18. Van Herck SLJ, Delbaere J, Bourgeois NMA, McAllan BM, Richardson SJ, Darras VM (2015) Expression of thyroid hormone transporters and deiodinases at the brain barriers in the embryonic chicken: Insights into the regulation of thyroid hormone availability during neurodevelopment. Gen Comp Endocrinol 214, 30-9.

19. Hulbert AJ (2000) Thyroid hormones and their effects: a new perspective. Biol Rev 75, 519-631.

20. Richardson SJ (2007) Cell and molecular biology of transthyretin and thyroid hormones. Int Rev Cytol 258, 137-93.

21. Yamauchi K, Ishihara A (2009) Evolutionary changes to tranthyretin: developmentally regulated and tissue-specific gene expression. FEBS $J$ 276, 5357-66.

22. Hamberger A, Nyström B, Silvenius H, Wikkelsø C (1990) The contribution from the choroid plexus and the periventricular CNS to amino acids and proteins in the human CSF. Neurochem Res 15, 307-12.

23. Schreiber G, Aldred AR, Jaworowski A, Nilsson C, Achen MG, Segal MB (1990) Thyroxine transport from blood to brain via transthyretin synthesis in choroid plexus. Am J Physiol Regul Integr Comp Physiol 258, R338-45.

24. Richardson SJ (2009) Evolutionary changes to transthyretin: evolution of transthyretin biosynthesis. FEBS J 276, 5342-56.

25. Hörnberg A, Eneqvist T, Olofsson A, Lundgren E, Sauer-Eriksson AE (2000) A comparative analysis of 23 structures of the amyloidogenic protein transthyretin. J Mol Biol 302, 649-69.

26. Kyte J, Doolittle RF (1982) A simple method for displaying the hydropathic character of a protein. J Mol Biol 157, 105-32.

27. Bradford MM (1976) A rapid and sensitive method for the quantitation of microgram quantities of pro- 
tein utilizing the principle of protein-dye binding. Anal Biochem 72, 248-54.

28. Twining SS (1984) Fluorescein isothiocyanate-labeled casein assay for proteolytic enzymes. Anal Biochem 143, 30-43.

29. Zhu YS, Kalyankar P, FitzGerald RJ (2015) Relative quantitation analysis of the substrate specificity of glutamyl endopeptidase with bovine $\alpha$-caseins. Food Chem 167, 463-7.

30. Swaisgood HE (1993) Review and update of casein chemistry. J Dairy Sci 76, 3054-61.

31. Thomas MJ, Bhat S, Sorci-Thomas MG (2008) Threedimensional models of HDL apoA-I: implications for its assembly and function. J Lipid Res 49, 1875-83.

32. Bashtovyy D, Jones MK, Anantharamaiah GM, Segrest JP (2011) Sequence conservation of apolipoprotein A-I affords novel insights into HDL structurefunction. $J$ Lipid Res 52, 435-50.

33. Remko M, Fitz D, Broer R, Rode BM (2011) Effect of metal ions $\left(\mathrm{Ni}^{2+}, \mathrm{Cu}^{2+}\right.$ and $\left.\mathrm{Zn}^{2+}\right)$ and water coordination on the structure of L-phenylalanine, L-tyrosine, L-tryptophan and their zwitterionic forms. $J \mathrm{Mol}$ Model 17, 3117-28.

34. Liao SM, Du QS, Meng JZ, Pang ZW, Huang RB (2013) The multiple roles of histidine in protein interactions. Chem Cent J 7, 44.

35. Popov IA, Starodubtseva NL, Indeikina MI, Kostyukevich YuI, Kononikhin AS, Nikolaeva MI, Kukaev EN, Kozin SA, et al (2013) Mass spectrometric identification of posttranslational modifications in transthyretin from human blood. Mol Biol 47, 885-93.

36. Letourneur O, Gervasi G, Gaïa S, Pagès J, Watelet B, Jolivet M (2001) Characterization of Toxoplasma gondii surface antigen 1 (SAG1) secreted from Pichia pastoris: evidence of hyper O-glycosylation. Biotechnol Appl Biochem 33, 35-45.

37. Zhang Q, Kelly JW (2003) Cys10 mixed disulfides make transthyretin more amyloidogenic under mildly acidic conditions. Biochemistry 42, 8756-61.

38. Henze A, Homann T, Serteser M, Can O, Sezgin O, Coskun A, Unsal I, Schweigert FJ, et al (2015) Posttranslational modifications of transthyretin affect the triiodonine-binding potential. J Cell Mol Med 19, 359-70.

39. Monaco HL (2002) Three-dimensional structure of the transthyretin-retinol-binding protein complex. Clin Chem Lab Med 40, 1229-36.

40. Palmieri LC, Lima LMTR, Freire JBB, Bleicher L, Polikarpov I, Almeida FCL, Foguel D (2010) Novel $\mathrm{Zn}^{2+}$-binding sites in human transthyretin: implications for amyloidogenesis and retinol-binding protein recognition. $J$ Biol Chem 285, 31731-41. 